\title{
Bispectral index monitoring correlates with the level of consciousness in brain injured patients
}

\author{
Jin Yong Jung, Cheol Beom Cho, and Bo Mi Min \\ Department of Anesthesiology and Pain Medicine, School of Medicine, Catholic University of Daegu, Daegu, Korea
}

Background: In general, measuring the level of consciousness in neurological patients is important. To assess the patient's mental status, the Glasgow Coma Scale (GCS) and the level of consciousness (drowsiness, stupor, and coma) have been used in clinical situations. The aim of the present study was to identify the correlation between bispectral index (BIS) and level of consciousness in brain injured patients.

Methods: Eighty-nine adult patients of both sexes were included in the study. A blinded observer evaluated the mental status (GCS and level of consciousness) of a patient who is admitted in intensive care unit with brain injury, while an investigator noted the patient's BIS. The BIS was measured using a BIS monitor, Model A-3000 vista ${ }^{\mathrm{TM}}$ with Sensor Bis quatro ${ }^{\mathrm{TM}}$ (Aspect Medical Systems, Norwood, USA). A Spearman's rank correlation coefficient was used to determine if the level of consciousness correlated with the BIS.

Results: In 89 patients, the BIS was found to be significantly correlated with the level of consciousness $(\mathrm{r}=0.723$, $\mathrm{P}<0.01)$ and GCS $(\mathrm{r}=0.646, \mathrm{P}<0.01)$. The BIS values increased with an increasing level of consciousness. Mean BIS values of coma, semicoma, stupor and drowsiness were $0.14 \pm 0.23,38.9 \pm 18.0,60.3 \pm 14.5$, and $73.6 \pm 16.5$, respectively.

Conclusions: In the present study, a significant correlation existed between level of consciousness and BIS. These findings suggest that BIS may be used for assessing the level of consciousness in brain injured patients. However, the scatter of BIS values for any level of consciousness limited the worth of BIS in predicting mentality except in coma patients. (Korean J Anesthesiol 2013; 64: 246-250)

Key Words: Bispectral index, Glasgow Coma Scale, Level of consciousness.

Received: July 4, 2012. Revised: September 17, 2012. Accepted: September 19, 2012.

Corresponding author: Jin Yong Jung, M.D., Department of Anesthesiology and Pain Medicine, School of Medicine, Catholic University of Daegu, 3056-6, Daemyung 4-dong, Nam-gu, Daegu 705-718, Korea. Tel: 82-53-650-4505, Fax: 82-53-650-4517, E-mail: jychung@cu.ac.kr (c) This is an open-access article distributed under the terms of the Creative Commons Attribution Non-Commercial License (http:// creativecommons.org/licenses/by-nc/3.0/), which permits unrestricted non-commercial use, distribution, and reproduction in any medium, provided the original work is properly cited. 


\section{Introduction}

The bispectral index (BIS) has been widely used in the operating room as a way to measure the hypnotic effects of drugs [1-5]. Recently, the use of BIS has been extended to intensive care unit (ICU) patients $[6,7]$. In general, it is important to measure the level of consciousness in neurosurgical patients. It is helpful to have a standard scale for measuring the level of consciousness in which the level of consciousness measured by one clinician matches the level of consciousness measured by a different clinician. In short, a consistent scoring system would minimize inter-observer differences. Such a scale would provide better outcomes for patients because health care personnel would be able to communicate a patient's neurological condition more precisely [8].

There are several methods to access neurologic function. The Richmond Agitation-Sedation Scale (RASS), the Riker SedationAgitation Scale (SAS), the Reaction Level Scale (RLS) and the Glasgow Coma Scale (GCS) are usually used for this purpose [911]. However, there is inherent inter-observer variability when using these scales. The level of consciousness is usually divided into 5 stages, i.e., alert, drowsy, stupor, semicoma, and coma. Drowsiness is a condition in which the patient remains in a light sleep but can be easily aroused by touch or noise and can remain alert for some time. Stupor defines a state in which the patient can be awakened only by vigorous and repeated stimuli. Semicoma entails a condition in which the patient responds to painful stimuli without spontaneous movement. Coma indicates a state from which the patient cannot be aroused by stimulation, and no purposeful attempt is made to avoid painful stimuli [12]. This classification is also used to determine mental status in the medical field with the other neurologic accessing methods such as the RASS, the SAS, the RLS and the GCS. However, this classification has the same problem as the others because the result measured by one clinician can be different from the result measured by another clinician.

The aims of the present study were to determine if the BIS score could be used to assess a patient's level of consciousness, to identify the range of BIS scores according to a patient's mental status and to assess whether a correlation existed between the BIS score and the level of consciousness in brain injured patients.

\section{Materials and Methods}

The study was conducted prospectively from August 2009 to January 2010 on all the successive patients admitted to the neurosurgical intensive care unit. After institutional approval, informed consents were obtained. Eighty-nine adult patients of both sexes were included in the study. We excluded patients who were on sedative medications, who would be operated on within 2 days with general anesthesia and who were less than 20 years of age.

A blinded observer evaluated the mental status (GCS and level of consciousness) of a patient, while an investigator measured the BIS in the same patient. The BIS was measured using the BIS monitor, Model A-3000 vista ${ }^{\mathrm{TM}}$ with Sensor Bis quatro $^{\mathrm{TM}}$ (Aspect Medical Systems, Norwood, USA). The sensor, which has four electrodes, was placed at an angle over the forehead. The first electrode was positioned at the midline, approximately $5 \mathrm{~cm}$ above the nose, and the second electrode was positioned just lateral and inferior to the first electrode. The third electrode was placed over the temporal region behind the angle of the eye, and the fourth electrode was positioned directly above and adjacent to the eyebrow. The BIS was measured for 5 min. The BIS score was included if the Signal Quality Index was above $75 \%$ and the electromyography score was below $50 \%$ [13]. Before the BIS measurement, we stimulated the patient to awaken from sleep. The mean BIS score was calculated by averaging the maximum BIS score and the minimum BIS score measured over $5 \mathrm{~min}$.

Correlations between the mean BIS score and the level of consciousness as well as GCS scores were evaluated using a Spearman's correlation test. BIS scores and GCS scores at different levels of consciousness were compared using one-way ANOVA with post hoc Bonferroni's test. All data are expressed as the mean $\pm \mathrm{SD}$. A P value $<0.05$ was considered statistically significant.

\section{Results}

The characteristics of enrolled patients are presented in Table 1. The average age of patients was 60 years and ranged from 29 to

Table 1. Characteristics of the Patients

\begin{tabular}{lc}
\hline Patient (no.) & 89 \\
Age & $60.4 \pm 14.3$ \\
Body weight (kg) & $61.6 \pm 10.1$ \\
Height (cm) & $163.9 \pm 8.4$ \\
NICU admission diagnosis, no. (M/F) & \\
Subarachnoid hemorrhage & $24(12 / 12)$ \\
Intracerebral hemorrhage & $23(17 / 6)$ \\
Subdural hemorrhage & $18(13 / 5)$ \\
Intraventricular hemorrhage & $5(4 / 1)$ \\
Epidural hemorrhage & $5(1 / 4)$ \\
Cerebral infarction & $4(2 / 2)$ \\
Cerebral contusion & $4(3 / 1)$ \\
Intracerebral aneurysm & $2(0 / 2)$ \\
Cerebellar hemorrhage & $1(0 / 1)$ \\
Hydrocephalus & $1(1 / 0)$ \\
Arteriovenous malformation & $1(1 / 0)$ \\
Osteomyelitis of vertebra & $1(0 / 1)$ \\
\hline
\end{tabular}

NICU: neurosurgical intensive care unit. 
86. The mean body weight was $61.6 \mathrm{~kg}$, and the mean height was $163.9 \mathrm{~cm}$. Most patients had intracranial mass lesions, such as a subarachnoid hemorrhage, intracerebral hemorrhage, and subdural hemorrhage. The level of consciousness ranged from coma $(n=14)$, semicoma $(n=10)$, stupor $(n=40)$, and drowsiness $(\mathrm{n}=25)$. There was a significant correlation between BIS scores and the level of consciousness ( $\mathrm{r}=0.726$; $\mathrm{P}<0.01)$ (Fig. 1). The GCS scores also showed a good correlation with BIS scores $(\mathrm{r}=$ 0.646; $\mathrm{P}<0.01$ ). However, the level of consciousness showed a better correlation with BIS scores than GCS scores when comparing correlation coefficient values. Mean BIS values, at different levels of consciousness, are shown in Table 2. There were significant differences among different levels of consciousness. The mean BIS values of coma, semicoma, stupor and drowsiness were $0.14 \pm 0.23,38.9 \pm 18.0,60.3 \pm 14.5$, and 73.6 \pm 16.5 , respectively. The BIS values increased with increasing levels of consciousness. The GCS scores of coma, semicoma, stupor and drowsiness were $3.5 \pm 1.1,5.0 \pm 1.3,8.6 \pm 2.2$, and

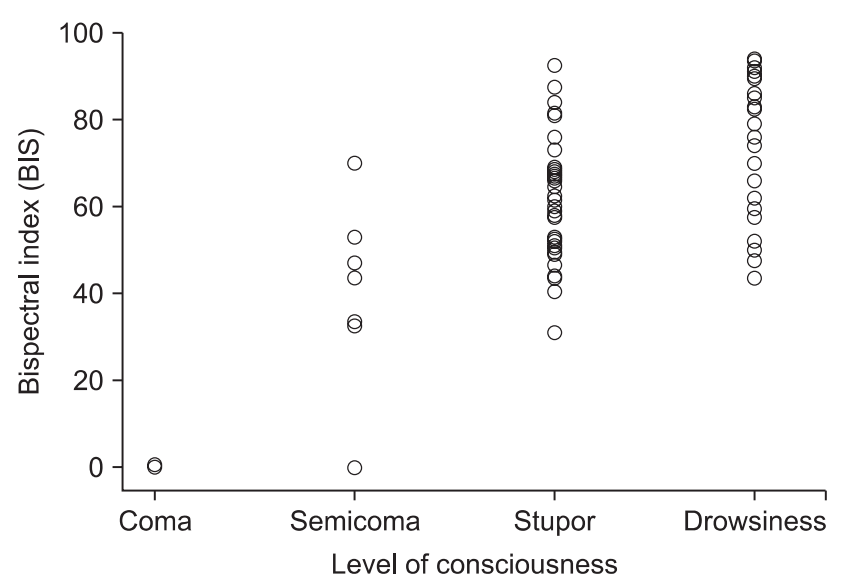

Fig. 1. Correlation between the level of consciousness and BIS for all data sets $(\mathrm{r}=0.726 ; \mathrm{P}<0.01)$.

Table 2. Mean BIS Values Corresponding to Various Levels of Consciousness

\begin{tabular}{llcc}
\hline \multirow{2}{*}{$\begin{array}{c}\text { Level of } \\
\text { consciousness }\end{array}$} & Mean BIS & \multicolumn{2}{c}{$95 \%$ Confidence interval } \\
\cline { 3 - 4 } & & $\begin{array}{c}\text { Lower } \\
\text { bound }\end{array}$ & $\begin{array}{c}\text { Upper } \\
\text { bound }\end{array}$ \\
\hline Coma $(\mathrm{n}=14)$ & $0.14 \pm 0.23$ & 0 & 0.3 \\
Semicoma $(\mathrm{n}=10)$ & $38.9 \pm 18.0^{*}$ & 26 & 51.8 \\
Stupor $(\mathrm{n}=40)$ & $60.3 \pm 14.5^{*, \dagger}$ & 55.7 & 65 \\
Drowsiness $(\mathrm{n}=25)$ & $73.6 \pm 16.5^{*, \dagger,}$ & 66.8 & 80.4
\end{tabular}

*Significant difference $(\mathrm{P}<0.001)$ compared to BIS values corresponding to Coma. ${ }^{\dagger}$ Significant difference $(\mathrm{P}<0.001)$ compared to $\mathrm{BIS}$ values corresponding to Semicoma. ${ }^{\ddagger}$ Significant difference $(\mathrm{P}<$ 0.001 ) compared to BIS values corresponding to Stupor. BIS values at different level of consciousnesswere compared by using one-way ANOVA with post hoc Bonferroni's test.
$11.8 \pm 1.8$, respectively (Table 3 ). There was no significant difference between coma and semicoma levels in terms of GCS scores. The GCS scores also increased with increasing levels of consciousness.

\section{Discussion}

This study was designed to determine the correlation between BIS scores and the level of consciousness in brain injured patients. We demonstrated that BIS scores showed good correlations with level of consciousness. When mental status decreased such as in a coma, there was a highly significant correlation with BIS scores. However, the BIS scores of semicoma, stupor, and drowsiness patients were much more scattered than those of coma patients. Although BIS scores were highly correlated with the level of consciousness, many patients experienced different mentalities that overlapped with the same BIS scores. We could only distinguish between coma patients from the other patients, using BIS monitoring, because coma patients' BIS scores ranged from 0 to 1 . The GCS also showed a good correlation with BIS scores. However, the level of consciousness had a higher correlation with BIS scores than GCS scores.

Results of the present study correspond well with those of earlier studies, which reported that a significant correlation exists between GCS and BIS in patients with mild to moderate head injuries [14]. These studies also demonstrated that for any GCS score, BIS scores were scattered, indicating the lack of a correlation between BIS and GCS. Schnakers et al. [15] reported that BIS most strongly correlated with the Glasgow Liege Scale, which comprises GCS with a quantified analysis of five brainstem reflexes, such as fronto-orbicular, vertical oculocephalic, papillary, horizontal oculo-cephalic, and oculocardiac. They suggested that BIS might be used to discriminate different clinical conditions in brain injured patients in the

Table 3. GCS Scores Corresponding to Various Levels of Consciousness

\begin{tabular}{|c|c|c|c|}
\hline \multirow{2}{*}{$\begin{array}{c}\text { Level of } \\
\text { consciousness }\end{array}$} & \multirow[b]{2}{*}{ GCS scores } & \multicolumn{2}{|c|}{ 95\% Confidence interval } \\
\hline & & $\begin{array}{l}\text { Lower } \\
\text { bound }\end{array}$ & $\begin{array}{l}\text { Upper } \\
\text { bound }\end{array}$ \\
\hline $\operatorname{Coma}(n=14)$ & $3.5 \pm 1.1$ & 2.9 & 4.1 \\
\hline Semicoma $(n=10)$ & $5.0 \pm 1.3$ & 4.0 & 6.0 \\
\hline Stupor $(n=40)$ & $8.6 \pm 2.2^{*, \dagger}$ & 7.9 & 9.3 \\
\hline Drowsiness $(n=25)$ & $11.8 \pm 1.8^{*, \dagger, キ}$ & 11.1 & 12.6 \\
\hline
\end{tabular}

*Significant difference $(\mathrm{P}<0.001)$ compared to GCS scores corresponding to Coma. ${ }^{\dagger}$ Significant difference $(\mathrm{P}<0.001)$ compared to GCS scores corresponding to Semicoma. ${ }^{\ddagger}$ Significant difference $(\mathrm{P}<$ 0.001) compared to GCS scores corresponding to Stupor. BIS values at different GCS scoreswere compared by using one-way ANOVA with post hoc Bonferroni's test. 
intensive care unit. The BIS was also useful in evaluating the consciousness level of unconscious children. Hsia et al. [16] demonstrated that a positive correlation exists between the GCS and BIS in critically ill children who were admitted to the pediatric intensive care unit. Cho et al. [17] demonstrated that BIS was significantly correlated with clinical sedation scales, such as RASS and RLS, in patients with brain injury.

However, Gill et al. [18] reported that BIS monitoring did not reliably correlate with GCS in emergency department patients. Although there was a significant difference between BIS and GCS in 38 patients, they noted a weak correlation (Spearman's rho $=0.387$ ). The problem with this weak correlation was the wide variability of BIS values corresponding to GCS. This finding was consistent with our results, showing that the BIS score could not predict the level of consciousness.

BIS is measured by the frontal lobe electrical activity processing raw electroencephalogram (EEG). Bispectral analysis is a signal processing methodology that assesses relationships among signal components and captures synchronization within signals, such as EEGs. By quantifying the correlation between all the frequencies within the signal, bispectral analysis yields an additional EEG facet of brain activity [19]. It is reported that a raw EEG can be useful for predicting outcome after a traumatic brain injury [20]. However, considerable expertise is needed to interpret a raw EEG. Therefore, BIS is a simple device that allows for continuous monitoring. The BIS was designed to measure the depth of anesthesia and sedation. It is interesting that BIS is calibrated for normal, anesthetized patients but not for brain injured patients. In addition, consciousness is maintained by the upper brainstem and thalamic neurons, the reticular activating system and its broad connection to the cerebral hemispheres [12]. Although BIS is an empirical, statistically derived variable that provides information about the interaction of brain cortical and subcortical regions [21], it cannot reflect subtle changes resulting from different brain injuries. Moreover, because BIS scores reflect only ipsilateral frontal lobe activity, where the BIS sensor is attached, it might be difficult to estimate function throughout the entire brain, although BIS scores correlate well with level of consciousness. This results in variable BIS values in patients with an unchanging level of consciousness and GCS.

When assessing level of consciousness and BIS, two scores are obtained from different brain regions that often do not correlate with each other. This lack of correlation is the primary limitation of our study. Assessing the level of consciousness and GCS may incorporate different elements of cerebral function into each score. In addition, BIS sensors used in the present study were attached to only one side of the frontal area, i.e., the left or right side. Thus, the BIS did not reflect the cerebral electrical activities of both frontal areas. Another limitation of the present study includes the different number of cases of each level of consciousness and sex because this study was performed under a given time constraint.

In conclusion, there was a significant correlation between BIS scores and the level of consciousness in brain injured intensive care unit patients. Although BIS scores could not precisely indicate a patient's mental status except in coma patients, it might be helpful for assessing the level of consciousness by utilizing BIS in conjunction with GCS or other neurological evaluation scales in brain injured patients. Further research is necessary to evaluate whether BIS can independently predict the exact level of consciousness.

\section{References}

1. Kim HM, Shin SW, Yoon JY, Lee HJ, Kim KH, Baik SW. Effects of etomidate on bispectral index scale and spectral entropy during induction of anesthesia by means of the raw electroencephalographic and electromyographic characteristics. Korean J Anesthesiol 2012; 62: 230-3.

2. Seol TK, Han MK, Lee HJ, Cheong MA, Jun JH. Bispectral index and their relation with consciousness of the patients who receive desflurane or sevoflurane anesthesia during wake-up test for spinal surgery for correction. Korean J Anesthesiol 2012; 62: 13-8.

3. Choi SS, Kim JS, Park IK, Lee G, Hahm KD. Changes in the bispectral index and cerebral oxygen saturation during neuroendovascular intervention under general anesthesia. Korean J Anesthesiol 2012; 62: 98-100.

4. Kang MH, In CB, Kim MH, Lim KJ, Park EY, Lee HM, et al. Inappropriate elevation of bispectral index values in robot assisted thyroidectomy with electromyographic endotracheal tube -A case report-. Korean J Anesthesiol 2011; 61: 511-4.

5. Bae JY, Choi do Y, Woo CH, Kwak IS, Mun SH, Kim KM. The BIS and hemodynamic changes in major burn patients according to a slow infusion of propofol for induction. Korean J Anesthesiol 2011; 60: 161-6.

6. Kwon MY, Lee SY, Kim TY, Kim DK, Lee KM, Woo NS, et al. Spectral entropy for assessing the depth of propofol sedation. Korean J Anesthesiol 2012; 62: 234-9.

7. Lee JI, Jun JH, Kim KS, Suh JK. Effect of intravenous propofol and fentanyl on bispectral index changes during endotracheal suction in ICU conscious patient. Korean J Anesthesiol 2007; 52: 156-60.

8. Rowley G, Fielding K. Reliability and accuracy of the Glasgow coma scale with experienced and inexperienced users. Lancet 1991; 337: 535-8.

9. Sessler CN, Gosnell MS, Grap MJ, Brophy GM, O'Neal PV, Tesoro EP, et al. The Richmond Agitation-Sedation Scale: validity and reliability in adult intensive care unit patients. Am J Respir Crit Care Med 2002; 166: 1338-44.

10. Riker RR, Fraser GL, Simmons LE, Wilkins ML. Validating the Sedation-Agitation Scale with the Bispectral Index and Visual Analog Scale in adult ICU patients after cardiac surgery. Intensive Care Med 2001; 27 : 853-8.

11. Walther SM, Jonasson U, Gill H. Comparison of the Glasgow Coma 
Scale and the Reaction Level Scale for assessment of cerebral responsiveness in the critically ill. Intensive Care Med 2003; 29: 9338.

12. Layon AJ, Gabrielli A, Friedman WA. Textbook of neurointensive care. Philadelphia, Elsevier Inc. 2004, pp 747-55.

13. Deogaonkar A, Gupta R, DeGeorgia M, Sabharwal V, Gopakumaran B, Schubert A, et al. Bispectral Index monitoring correlates with sedation scales in brain-injured patients. Crit Care Med 2004; 32: 2403-6.

14. Paul DB, Umamaheswara Rao GS. Correlation of Bispectral Index with Glasgow Coma Score in mild and moderate head injuries. J Clin Monit Comput 2006; 20: 399-404.

15. Schnakers C, Majerus S, Laureys S. Bispectral analysis of electroencephalogram signals during recovery from coma: preliminary findings. Neuropsychol Rehabil 2005; 15: 381-8.
16. Hsia SH, Wu CT, Wang HS, Yan DC, Chen SC. The use of bispectral index to monitor unconscious children. Pediatr Neurol 2004; 31: 20-3.

17. Cho JH, Cheong SH, Kim HS, Kim SH, Cho KR, Lee SE, et al. Bispectral index monitoring to assess the level of consciousness in patients with brain injury. Korean J Anesthesiol 2009; 57: 185-9.

18. Gill M, Green SM, Krauss B. Can the bispectral index monitor quantify altered level of consciousness in emergency department patients? Acad Emerg Med 2003; 10: 175-9.

19. Sigl JC, Chamoun NG. An introduction to bispectral analysis for the electroencephalogram. J Clin Monit 1994; 10: 392-404.

20. Zandbergen EG, de Haan RJ, Stoutenbeek CP, Koelman JH, Hijdra A. Systematic review of early prediction of poor outcome in anoxicischaemic coma. Lancet 1998; 352: 1808-12.

21. Rampil IJ. A primer for EEG signal processing in anesthesia. Anesthesiology 1998; 89: 980-1002. 